\title{
SOME SHARP INEQUALITIES FOR MARTINGALE TRANSFORMS
}

\author{
K. P. CHOI
}

\begin{abstract}
Two sharp inequalities for martingale transforms are proved. These results extend some earlier work of Burkholder. The inequalities are then extended to stochastic integrals and differentially subordinate martingales.
\end{abstract}

1. Introduction. A gambler starting with an initial fortune $\alpha>0$ plays a sequence of fair games (in the sense of a martingale to be explained in the next section). Let $\beta>\alpha$. Can he be assured that he can increase his fortune to $\beta$ without running into debt?

This was answered negatively by Ville [6]. Indeed, he showed that if $f=$ $\left(f_{1}, f_{2}, \ldots\right)$ is a nonnegative martingale starting at $\alpha$, that is $f_{1} \equiv \alpha$, then

$$
P\left(\sup _{n \geq 1} f_{n} \geq \beta\right) \leq \frac{\alpha}{\beta}
$$

This inequality can be seen to be sharp: the number $\alpha / \beta$ on the right cannot be replaced by any smaller number independent of $f$. Inequality (1.1) was recently extended by Burkholder [4] to

$$
P\left(\sup _{n \geq 1} g_{n} \geq \beta\right) \leq \frac{\alpha}{\beta}
$$

where $g=\left(g_{1}, g_{2}, g_{3}, \ldots\right)$ is the martingale transform of a nonnegative martingale $f$ by a predictable sequence $\theta=\left(\theta_{1}, \theta_{2}, \ldots\right)$, each term $\theta_{k}$ having its values in $[0,1]$. (For definitions, see §2.) In other words, a gambler with an initial fortune $\alpha>0$ cannot improve his chance beyond $\alpha / \beta$ of increasing his fortune to $\beta$ without going into debt even if he has a certain control of the martingale: he can either skip the $n$th game or play a smaller bet than he otherwise would have had to based on the outcomes of the previous $n-1$ games.

The classical weak type inequality of Doob [5] for real-valued martingales (not necessarily nonnegative and with $\|f\|_{1}$ and $f^{*}(\omega)$ denoting $\sup _{n \geq 1}\left\|f_{n}\right\|_{1}$ and

Received by the editors November 7, 1986 and, in revised form, March 30, 1987.

1980 Mathematics Subject Classification (1985 Revision). Primary 60G42, 60H05; Secondary $60 \mathrm{G} 46$.

Key words and phrases. Martingale, martingale transform, nonlinear partial differential equation, differential subordination, stochastic integrals.

This paper constitutes a portion of the author's Ph.D. dissertation written under Professor Donald L. Burkholder at the University of Illinois. The author wishes to express his thanks to Professor Burkholder for suggesting this problem and his support and encouragement during the course of this work. 
$\sup _{n \geq 1}\left|f_{n}(\omega)\right|$ respectively),

$$
\lambda P\left(\sup _{n \geq 1}\left|f_{n}\right| \geq \lambda\right) \leq\|f\|_{1}, \quad \lambda>0,
$$

contains Ville's inequality (1.1): If $f$ is a nonnegative martingale, then $\|f\|_{1}=$ $E f_{1}=\alpha$.

Doob's inequality can also be extended to martingale transforms. Burkholder shows in [4], that if $g$ is the transform of a real martingale $f$ by a predictable sequence $v=\left(v_{1}, v_{2}, \ldots\right)$, each term $v_{k}$ having its values in $[0,1]$, then

$$
\lambda P\left(g^{*} \geq \lambda\right) \leq\|f\|_{1}, \quad \lambda>0 .
$$

The proof of (1.4) differs greatly from the proof of (1.3) and inequalities (1.2) and (1.4) are perhaps surprising since $g$ need not be $L^{1}$-bounded. If the predictable sequence $v=\left(v_{1}, v_{2}, \ldots\right)$ satisfies $a \leq v_{k}(\omega) \leq b$, where $a \leq 0 \leq b$, then the above inequality becomes (see [4])

$$
\lambda P\left(g^{*} \geq \lambda\right) \leq(b-a)\|f\|_{1}, \quad \lambda>0,
$$

and the constant $b-a$ on the right is sharp.

Let $\alpha, \beta \in \mathbf{R}$. If a gambler with initial fortune $\alpha$ is allowed to control his martingale $f$ by a predictable sequence $v=\left(1, v_{2}, v_{3}, \ldots\right)$, each term $v_{k}$ having its. values in $[-1,1]$ so that the transform $g$ has at least probability $t$ of exceeding $\beta$, then how small can $\|f\|_{1}$ be? This is answered in the following theorem, one of our main results.

THEOREM 1.1. Let $\alpha, \beta \in \mathbf{R}$ and $t \in[0,1]$. Let $f=\left(f_{1}, f_{2}, \ldots\right)$ be a real martingale with $f_{1} \equiv \alpha$. If $g$ is the transform of $f$ by a predictable sequence $v=$ $\left(1, v_{2}, \ldots\right)$, with $v_{k}$ having its values in $[-1,1]$ for all $k \geq 2$, and $g$ satisfies the one-sided condition:

$$
P\left(\sup _{n \geq 1} g_{n} \geq \beta\right) \geq t
$$

then

$$
\|f\|_{1} \geq|\alpha| \vee\left\{\beta-\alpha-\left[\beta^{+}(\beta-2 \alpha)^{+}(1-t)\right]^{1 / 2}\right\}
$$

and this inequality is sharp.

Here $x^{+}=x \vee 0$, the maximum of $x$ and 0 .

In fact, the lower bound of (1.6) can be attained by some real martingale $f$ and $g$ its transform by the constant sequence $v \equiv(1,-1,1,-1, \ldots)$.

The special case of Theorem 1.1 when $t=1$ is due to Burkholder (see Theorem 7.3 in [3]).

In the proof of Theorem 1.1 we need the following closely related result which is of independent interest.

THEOREM 1.2. Let $x, y \in \mathbf{R}$ and $t \in[0,1]$. Let $f=\left(f_{1}, f_{2}, \ldots\right)$ be a real martingale starting at $(x+y) / 2$. If $g$ is the transform of $f$ by a predictable sequence $v=\left(1, v_{2}, \ldots\right)$, with $v_{k}$ having its values in $[-1,1]$ for all $k \geq 2$ and $g$ satisfies the two-sided condition:

$$
P\left(\sup _{n \geq 1}\left|g_{n}-y\right| \geq 1\right) \geq t
$$


then

$$
\|f\|_{1} \geq \frac{1}{2} L(x, y, t)
$$

where $L(x, y, t)$ is defined in $§ 3$. Inequality (1.7) is sharp.

Again, the lower bound of (1.7) can already be attained by some real martingale $f$ and $g$ its transform by the constant sequence $v \equiv(1,-1,1,-1, \ldots)$.

The special case when $t=1$ has also been found by Burkholder (see Remark 7.1 in [3]). The gambling interpretation of Theorem 1.2 is similar to that of Theorem 1.1. Also, see Theorem 3.2 of [2] for a version of Theorem 1.2 in the case $f$ is Banach-spaced valued. Our theorem gives the optimal function $\Lambda$ of that theorem in the real case.

The proofs of Theorems 1.1 and 1.2 hinge on functions satisfying certain convexity conditions and boundary conditions. They are found by solving a system of partial differential equations and inequalities much in the spirit of the method in [3]. This will be explained in $\S 4$.

Inequalities (1.6) and (1.7) carry over to stochastic integrals: Let $\left(\Omega, \mathcal{F}_{\infty}, P\right)$ be a complete probability space and $\mathcal{F}=\left(\xi_{t}\right)_{t \geq 0}$ a nondecreasing right-continuous family of sub- $\sigma$-fields of $\xi_{\infty}$ where $\xi_{0}$ contains all of the sets $A$ in $\xi_{\infty}$ with $P(A)=0$. Let $V=\left(V_{t}\right)_{t \geq 0}$ be a real predictable process uniformly bounded in absolulte value by 1 . Assume that $X=\left(X_{t}\right)_{t \geq 0}$ is a real martingale adapted to $\mathcal{F}$ and that almost all of the paths of $X$ are right continuous on $[0, \infty)$ with left limits $(0, \infty)$. Define

$$
Y_{t}=\int_{[0, t]} V d X \quad \text { a.s. }
$$

THEOREM 1.3. Let $\alpha, \beta \in \mathbf{R}, t \in[0,1]$. Suppose that $Y$ is a stochastic integral of $V$ with respect to $X$ as above with $X_{0} \equiv \alpha$ and

$$
P\left(\sup _{t \geq 0} Y_{t} \geq \beta\right) \geq t
$$

then

$$
\sup _{t \geq 0} E\left|X_{t}\right|=\|X\|_{1} \geq|\alpha| \vee\left\{\beta-\alpha-\left[\beta^{+}(\beta-2 \alpha)^{+}(1-t)\right]^{1 / 2}\right\} .
$$

This inequality is, of course, sharp since it is sharp in the discrete case.

We also have a similar extension of Theorem 1.2 to stochastic integrals.

Another extension of Theorems 1.1 and 1.2 is to the case of differentially subordinate martingales. Indeed, if $(f, g)=\left\{\left(f_{n}, g_{n}\right): n \geq 1\right\}$ is a martingale starting at $((x+y) / 2,(x+y) / 2)$ with $g$ differentially subordinate to $f$ satisfying the two-sided condition (S2), then (1.7) holds for $f$. We have a similar extension of Theorem 1.1. For the method of proof of these extensions, see [3].

2. Definitions and notations. Let us recall some definitions and fix notations.

A sequence $d=\left(d_{1}, d_{2}, \ldots\right)$ of real-valued integrable functions on a probability space is said to be a martingale difference sequence if for every bounded continuous function $\varphi: \mathbf{R}^{n} \rightarrow \mathbf{R}$ we have

$$
E \varphi\left(d_{1}, d_{2}, \ldots, d_{n}\right) d_{n+1}=0 \text { for all } n \geq 1 \text {. }
$$

The sequence $f=\left(f_{1}, f_{2}, \ldots\right)$ of the partial sums $f_{n}=\sum_{k=1}^{n} d_{k}$ is then a martingale. This is, of course, equivalent to the usual definition [3]. 
A sequence of measurable functions $v=\left(v_{1}, v_{2}, \ldots\right)$ is predictable (relative to the martingale $f$ ) if for every $n \geq 1$ and every bounded continuous function $\varphi: \mathbf{R}^{2 n+1} \rightarrow \mathbf{R}$ we have

$$
E \varphi\left(d_{1}, \ldots, d_{n}, v_{1}, \ldots, v_{n+1}\right) d_{n+1}=0 .
$$

A sequence $g=\left(g_{1}, g_{2}, \ldots\right)$ is the transform of $f$ by $v=\left(v_{1}, v_{2}, \ldots\right)$ if, for every $n \geq 1$,

$$
g_{n}=\sum_{k=1}^{n} v_{k} d_{k} .
$$

Indeed, $g$ is also a martingale if $v_{k} d_{k}$ is integrable, $k \geq 1$, as it is if the sequence $v$ is uniformly bounded.

A very important special case of martingale transforms arise when each term of the sequence $v=\left(v_{1}, v_{2}, \ldots\right)$ takes values in $\{1,-1\}$.

These definitions can be extended to martingales with values in $\mathbf{R}^{2}$ or, indeed, any Banach space.

Throughout this paper, we will use $[a, b)$, when there is no risk of ambiguity, to denote either the interval $\{x: a \leq x<b\}$ or the indicator function of this interval.

We will also use $\bar{\Omega}$ to denote the closure of a set $\Omega$.

3. Proofs of Theorems 1.1 and 1.2. Before we proceed to the proofs of Theorems 1.1 and 1.2 , we need the following definitions. Let

$$
\begin{aligned}
D & =\mathbf{R} \times \mathbf{R} \times(0,1), \\
D_{1} & =\{(x, y, t) \in D: x+y>0 \text { and } x+y>(1+y) t\}, \\
D_{2} & =\{(x, y, t) \in D: x+y>0 \text { and } x+y<(1+y) t\}, \\
D_{3} & =\left\{(x, y, t) \in D:(-y,-x, t) \in D_{2}\right\}, \\
D_{4} & =\left\{(x, y, t) \in D:(-y,-x, t) \in D_{1}\right\} .
\end{aligned}
$$

Define $L_{1}: \mathbf{R} \times \mathbf{R} \times[0,1] \rightarrow \mathbf{R}$ to be the continuous function satisfying

$$
L_{1}(x, y, t)=L_{1}(-y,-x, t)
$$

and

$$
L_{1}(x, y, t)= \begin{cases}x+y & \text { on } D_{1} \\ 2+y-x-2[(1-x)(1+y)(1-t)]^{1 / 2} & \text { on } D_{2}\end{cases}
$$

Let

$$
\begin{aligned}
\Omega= & \mathbf{R} \times \mathbf{R} \times(0,1) \\
\Omega_{1}^{\prime}= & \{(x, y, t) \in \Omega: x \leq 0, x+y>0 \text { and } x+y>(1+x) t\}, \\
\Omega_{1}^{\prime \prime}= & \{(x, y, t) \in \Omega: 0<x \leq 1,0<y \leq 1 \text { and }(1-y) x+y>t\} \\
& \cup\{(x, y, t) \in \Omega: x \vee y>1\}, \\
\Omega_{1}^{\prime \prime \prime}= & \{(x, y, t) \in \Omega: 0 \geq y, x+y>0 \text { and } x+y>(1+y) t\} \\
\Omega_{1}= & \Omega_{1}^{\prime} \cup \Omega_{1}^{\prime \prime} \cup \Omega_{1}^{\prime \prime \prime} \\
\Omega_{2}= & \{(x, y, t) \in \Omega:-(1-x) t<x+y<(1+y) t \text { and } t<(1+y) x-y\}, \\
\Omega_{3}= & \left\{(x, y, t) \in \Omega:(-x,-y, t) \in \Omega_{1}\right\} \\
\Omega_{4}= & \left\{(x, y, t) \in \Omega:(-x,-y, t) \in \Omega_{2}\right\} \\
\Omega_{5}= & \{(x, y, t) \in \Omega:(1-|y|)|x|+|y|<t\} .
\end{aligned}
$$


Since $0<t<1$, the set $\Omega_{i}$ is open for $1 \leq i \leq 5$.

Define $L: \mathbf{R} \times \mathbf{R} \times[0,1] \rightarrow \mathbf{R}$ to be the continuous function satisfying

$$
L(x, y, t)=L(-x,-y, t)=L(y, x, t)
$$

and

$$
L(x, y, t)= \begin{cases}x+y & \text { on } \Omega_{1} \\ t+x y & \text { on } \Omega_{5} \\ 2+y-x-2[(1-x)(1+y)(1-t)]^{1 / 2} & \text { on } \Omega_{2}\end{cases}
$$

It can be verified readily that $L_{1}$ and $L$ are both well defined and continuous.

PROOF OF THEOREM 1.1. There are two parts in the proof. In Part I, we use (1.7) to derive

$$
\|f\|_{1} \geq \frac{1}{2} L_{1}(x, y, t)
$$

and we show that, with the change of variables

$$
(\alpha, \beta)=((x+y) / 2, y+1),
$$

(3.5) and (1.6) are equivalent. Then, in Part II, we prove that (3.5) is sharp by examples.

Part I. Let $\mathcal{M}(x, y, t)$ denote the class of all real martingales $f$ such that there exists a transform $g$ of $f$ by a predictable sequence $v=\left(1, v_{2}, v_{3}, \ldots\right)$ with values in $[-1,1]$ satisfying the one-sided condition (S1).

Define

$$
x_{j}=\frac{2 x}{j+1}+\frac{j-1}{j+1} \text { and } y_{j}=\frac{2 y}{j+1}-\frac{j-1}{j+1} .
$$

Following the same lines of proof of Theorem 7.3 of $[\mathbf{3}]$, we have: If $f \in \mathcal{M}(x, y, t)$, then

$$
\|f\|_{1} \geq \lim _{j \rightarrow \infty} \frac{j+1}{4} L\left(x_{j}, y_{j}, t\right) .
$$

Therefore (3.5) will be proved if we can show that, if $(x, y, t) \in \mathbf{R} \times \mathbf{R} \times(0,1)$, then

$$
L_{1}(x, y, t)=\lim _{j \rightarrow \infty} \frac{j+1}{2} L\left(x_{j}, y_{j}, t\right) .
$$

To show (3.7), we have three cases: (i) $(x, y, t) \in D_{1}$, (ii) $(x, y, t) \in D_{2}$ and (iii) $(x, y, t) \in D_{3} \cup D_{4}$. All the other cases follow at once from these three.

Case (i). $(x, y, t) \in D_{1}$. Now choose $j$ large enough such that $y<(j-1) / 2$, then $y_{j}<0, x_{j}+y_{j}=2(x+y) /(j+1)>0$ and

$$
x_{j}+y_{j}=\frac{2}{j+1}(x+y)>\frac{2}{j+1}(1+y) t=\left(1+y_{j}\right) t .
$$

Therefore $\left(x_{j}, y_{j}, t\right) \in \Omega_{1}^{\prime \prime \prime}$ for $j$ large,

$$
\lim _{j \rightarrow \infty} \frac{j+1}{2} L\left(x_{j}, y_{j}, t\right)=\lim _{j \rightarrow \infty} \frac{j+1}{2}\left(x_{j}+y_{j}\right)=x+y=L_{1}(x, y, t) .
$$

Case (ii). $(x, y, t) \in D_{2}$. Since $t<1$ and $\lim _{j \rightarrow \infty}\left(1+y_{j}\right) x_{j}-y_{j}=1$, choose $j$ so large that $t<\left(1+y_{j}\right) x_{j}-y_{j}$. Also

$$
-\left(1-x_{j}\right) t=\frac{-2(1-x) t}{j+1}<\frac{2}{j+1}(x+y)=x_{j}+y_{j}<\frac{2}{j+1}(1+y) t=\left(1+y_{j}\right) t .
$$


That is, $\left(x_{j}, y_{j}, t\right) \in \Omega_{2}$ for $j$ large enough. Therefore

$$
\begin{aligned}
\lim _{j \rightarrow \infty} \frac{j+1}{2} L\left(x_{j}, y_{j}, t\right) & =\lim _{j \rightarrow \infty} \frac{j+1}{2}\left\{2+y_{j}-x_{j}-2\left[\left(1-x_{j}\right)\left(1+y_{j}\right)(1-t)\right]^{1 / 2}\right\} \\
& =2+y-x-2[(1-x)(1+y)(1-t)]^{1 / 2} \\
& =L_{1}(x, y, t) .
\end{aligned}
$$

Case (iii). Our remaining case. If $(x, y, t) \in D_{3} \cup D_{4}$ then $(-y,-x, t) \in D_{1} \cup D_{2}$, let $(X, Y)=(-y,-x)$ and $X_{j}, Y_{j}$ similarly defined, then $\left(X_{j}, Y_{j}\right)=\left(-y_{j},-x_{y}\right)$ and

$$
\begin{aligned}
\lim _{j \rightarrow \infty} \frac{j+1}{2} L\left(x_{j}, y_{j}, t\right) & =\lim _{j \rightarrow \infty} \frac{j+1}{2} L\left(-y_{j},-x_{j}, t\right) \quad \text { by }(3.3) \\
& =\lim _{j \rightarrow \infty} \frac{j+1}{2} L\left(X_{j}, Y_{j}, t\right) \\
& =L_{1}(X, Y, t) \quad \text { by Cases (i) and (ii) } \\
& =L_{1}(-Y,-X, t) \quad \text { by (3.1) } \\
& =L_{1}(x, y, t) .
\end{aligned}
$$

This completes the proof of (3.7).

It is straightforward to check that on $D_{1}$

$$
|x+y| \geq(1+y)+(1-x)-2\left[(1-x)^{+}(1+y)^{+}(1-t)\right]^{1 / 2}
$$

and this inequality is reversed on $D_{2}$. That is,

$$
\begin{aligned}
\frac{1}{2} L_{1}(x, y, t) & =\frac{|x+y|}{2} \vee\left\{\frac{2+y-x-2\left[(1-x)^{+}(1+y)^{+}(1-t)\right]^{1 / 2}}{2}\right\} \\
& =|\alpha| \vee\left\{\beta-\alpha-\left[\beta^{+}(\beta-2 \alpha)^{+}(1-t)\right]^{1 / 2}\right\}
\end{aligned}
$$

with the change of variables (3.6). Using (3.5), we obtain (1.6).

Part II. To show that (3.5) is sharp, we consider seven cases, namely: (i) $y+2<x$; (ii) $y+2>x$ and $y \leq-1$; (iii) $y+2>x$ and $x \geq 1$; (iv) $y>-1, x<1$ and $0 \leq t \leq(x+y) /(1+y)$; (v) $y>-1, x<1$ and $0 \leq(x+y) /(1+y) \leq t$; (vi) $y>-1$, $x<1, x+y<0$ and $0 \leq t \leq-(x+y) /(1-x)$ and (vii) $y>-1, x<1, x+y<0$ and $-(x+y) /(1-x)<t \leq 1$. For the first three cases, by (3.6), they correspond to $\beta \leq \alpha, \alpha<\beta \leq 0$ and $0<\frac{1}{2} \beta \leq \alpha<\beta$, which are identical to Burkholder's first three cases in the proof of Theorem 7.3 of [3].

Case (iv). We may assume $x+y>0$, otherwise there is nothing to prove. Let

$$
f_{1}=\frac{x+y}{2}[0,1), \quad f_{n}=2^{n-3}(y+1)\left[0, \frac{x+y}{2^{n-2}(y+1)}\right)
$$

for all $n \geq 2$. Now $f$ is a nonnegative martingale and

$$
P\left(\sup _{n \geq 1} g_{n} \geq \beta\right)=\frac{x+y}{1+y} \geq t,
$$

where $g$ is the transform of $f$ by $v=(1,1,-1,-1,-1, \ldots)$,

$$
\|f\|_{1}=\left\|f_{1}\right\|_{1}=\frac{x+y}{2}=\frac{1}{2} L_{1}(x, y, t) .
$$


Case (vi) is similar. Let $0<\gamma=-(x+y) /(1-x)<1, f_{1}=((x+y) / 2)[0,1)$, $f_{n}=-2^{n-3}(1-x)\left[0, \gamma / 2^{n-2}\right)$ for all $n \geq 2$. Note that $f$ is a nonpositive martingale. Let $g$ be the transform of $f$ by $v=(1,-1,1,1,1, \ldots)$; then

$$
P\left(\sup _{n \geq 1} g_{n} \geq \beta\right)=\gamma=-\frac{x+y}{1-x} \geq t
$$

and

$$
\|f\|_{1}=-E f_{1}=-\frac{x+y}{2}=\frac{1}{2} L_{1}(x, y, t) .
$$

Case (v) and Case (vii) are similar, and we will only work out Case (v) in detail.

Case (v). We may also assume $0<t<1$ : for $t=1$ has already been treated in Theorem 7.3 in [3] and for $t=0,(3.5)$ is obvious. Therefore $0<1-t<$ $(1-x) /(1+y)$. Let

$$
0<\mu=\lambda=\left[\frac{(1+y)(1-t)}{1-x}\right]^{1 / 2}<1 .
$$

Since $x+y \geq 0$, we have $1+y \geq 1-x>(1-x)(1-t)$. Thus

$$
0<\gamma=1-\left[\frac{(1-x)(1-t)}{1+y}\right]^{1 / 2}<1 \text {. }
$$

Let $a_{0}=\gamma+\mu(1-\gamma)$ and for all $n \geq 1, a_{n}=\left(2^{n}-1+a_{0}\right) / 2^{n}$. Let $f_{1} \equiv(x+y) / 2$,

$$
f_{2}=\frac{1+y}{2}[0, \gamma)-\frac{(1-\lambda)(1+y)}{2 \lambda}[\gamma, 1)
$$

and for every $n \geq 3$,

$$
f_{n}=2^{n-3}(1+y)\left[0, \frac{1}{2^{n-2}} \gamma\right)-2^{n-4} \frac{1+y}{\lambda}\left[a_{n-3}, 1\right) .
$$

It is not difficult to see that $f$ is a martingale. For $0 \leq s<\gamma$, let $v=\varepsilon^{\prime}$, and for $\gamma \leq$ $s<1$, let $v=\varepsilon^{\prime \prime}$ where $\varepsilon^{\prime}=(1,1,-1,-1,-1, \ldots)$ and $\varepsilon^{\prime \prime}=(1,1,-1,1,1,1, \ldots)$. By construction, $v$ is predictable relative to $f$. It is not difficult to verify that

$$
\left\{s \in[0,1): \sup _{n \geq 1} g_{n} \geq \beta\right\}=[0, \gamma) \cup\left[a_{0}, 1\right) \text {. }
$$

Therefore,

$$
P\left(\sup _{n \geq 1} g_{n} \geq \beta\right)=\gamma+1-a_{0}=1-\mu(1-\gamma)=1-(1-t)=t .
$$

Now

$$
\begin{aligned}
\|f\|_{1} & =\sup _{n \geq 1} E\left|f_{n}\right|=\lim _{n \rightarrow \infty} \frac{(1+y) \gamma}{2}+\frac{2^{n-4}(1+y)}{\lambda}\left(1-a_{n-3}\right) \\
& =\frac{(1+y) \gamma}{2}+\frac{1}{2}\left[\frac{(1-x)(1+y)}{1-t}\right]^{1 / 2}(1-\gamma)(1-\mu) \\
& =\frac{2+y-x-2[(1-x)(1+y)(1-t)]^{1 / 2}}{2} \\
& =\frac{1}{2} L_{1}(x, y, t) .
\end{aligned}
$$


This finishes the proof of Theorem 1.1.

Examining the examples in Part II of the above proof, we see that $v_{k} \in\{1,-1\}$ in each case. Now using the technique in Lemma 2.1 in $[3]$, we have the remark immediately after Theorem 1.1.

We collect below properties of $L(x, y, t)$, which will be used in the proof of Theorem 1.2, in the following lemma.

LEMMA 3.1. (a) There exists a positive real number $M$ such that $-M \leq$ $L(x, y, t)-|x+y| \leq M$ for all $(x, y, t) \in \mathbf{R} \times \mathbf{R} \times[0,1]$.

(b) $L(x, y, 0)=|x+y|,(x, y) \in \mathbf{R}^{2}$.

(c) $L(x, y, 1)=|x+y|,|x| \vee|y| \geq 1$.

(d) For $h, k, r \in \mathbf{R}$ with $h k \geq 0$, we have

$$
\begin{aligned}
& L(x+h s, y+k s, t+r s) \\
& \quad \geq L(x, y, t)+h s \varphi_{1}(x, y, t)+k s \varphi_{2}(x, y, t)+r s \varphi_{3}(x, y, t)
\end{aligned}
$$

for all $s \in \mathbf{R}$ such that $t+r s \in[0,1]$, and where $\varphi_{i}, 1 \leq i \leq 3$, are bounded Borel measurable functions.

We will prove this lemma in $\S 5$.

PROOF OF THEOREM 1.2. We divide the proof into two parts. We establish (1.7) in Part I and in Part II we show that (1.7) is sharp.

Part I. Let $\mathcal{N}(x, y, t)$ be the class of all real martingales $f$ starting at $(x+y) / 2$ such that there exists a transform, $g$, of $f$ by a predictable sequence $v=\left(1, v_{2}, v_{3}, \ldots\right)$ with values in $[-1,1]$ satisfying the two-sided condition (S2). We observe that $\mathcal{M}(x, y, t) \subseteq \mathcal{N}(x, y, t)$.

Let $f \in \mathcal{N}(x, y, t)$. Let $v$ and $g$ be as associated with $f$ in the definition of $\mathcal{N}(x, y, t)$. Let $A_{n}=\sigma\left(f_{1}, f_{2}, \ldots, f_{n}\right)$ for all $n \geq 1$. In particular, $A_{1}$ is the trivial $\sigma$-field. Let $A_{\infty}=\bigvee_{n=1}^{\infty} A_{n}$ be the smallest $\sigma$-field containing $A_{n}$ for all $n \geq 1$. We have

$$
P\left(\sup _{n \geq 1}\left|g_{n}-y\right| \geq 1\right)=t^{\prime} \geq t .
$$

Define $\left(X_{1}, Y_{1}, T_{1}\right)=\left(x, y, t^{\prime}\right)$ and, for all $n \geq 2$,

$$
\begin{aligned}
& X_{n}=x+\sum_{k=2}^{n}\left(1+v_{k}\right) d_{k}, \\
& Y_{n}=y+\sum_{k=2}^{n}\left(1-v_{k}\right) d_{k},
\end{aligned}
$$

and

$$
T_{n}=E\left(1\left\{\sup _{m \geq 1}\left|g_{m}-y\right| \geq 1\right\} \mid A_{n}\right) \text {. }
$$

Then $W=\left\{W_{n}=\left(X_{n}, Y_{n}, T_{n}\right): n \geq 1\right\}$ is a $\mathbf{R}^{3}$-valued martingale starting at $\left(x, y, t^{\prime}\right)$. Indeed, $W_{n} \in \mathbf{R} \times \mathbf{R} \times[0,1]$ a.e. By (3.8), we obtain

$$
\begin{aligned}
L\left(W_{n+1}\right) \geq & L\left(W_{n}\right)+\left(1+v_{n+1}\right) d_{n+1} \varphi_{1}\left(W_{n}\right)+\left(1-v_{n+1}\right) d_{n+1} \varphi_{2}\left(W_{n}\right) \\
& +\left(T_{n+1}-T_{n}\right) \varphi_{3}\left(W_{n}\right) .
\end{aligned}
$$


Indeed, when $d_{n+1} \neq 0$, let $h=\left(1+v_{n+1}\right) \geq 0, k=\left(1-v_{n+1}\right) \geq 0, r=$ $\left(T_{n+1}-T_{n}\right) / d_{n+1}$ and $s=d_{n+1}$ in (3.8), then (3.12) follows; when $d_{n+1}=0$, let $h=k=0, r=T_{n+1}-T_{n}$ and $s=1$, then (3.12) follows from (3.8).

Now

$$
E\left(1+v_{n+1}\right) d_{n+1} \varphi_{1}\left(W_{n}\right)=E\left(\left(1+v_{n+1}\right) \varphi_{1}\left(W_{n}\right) E\left(d_{n+1} \mid A_{n}\right)\right)=0 .
$$

Similarly,

$$
E\left(1-v_{n+1}\right) d_{n+1} \varphi_{2}\left(W_{n}\right)=E\left(T_{n+1}-T_{n}\right)=0 .
$$

Therefore, after taking expectations of both sides of (3.12), we obtain

$$
E L\left(W_{n+1}\right) \geq E L\left(W_{n}\right) \geq \cdots \geq E L\left(W_{1}\right)=L\left(x, y, t^{\prime}\right) .
$$

Without loss of generality, we may assume $\|f\|_{1}<\infty$, otherwise (1.7) is trivial. Since $f$ is $L^{1}$-bounded, then by the martingale convergence theorem, $f_{n} \rightarrow f_{\infty}$ a.e. Also $g_{n} \rightarrow g_{\infty}$ a.e. by Theorem 1 of $[\mathbf{1}]$. We may also assume that $T_{\infty}=1 \mathrm{implies}$ $\left|Y_{\infty}\right| \geq 1$, otherwise, we stop $W$ at the first time $\left|g_{n}-y\right| \geq 1$ without increasing the $L^{1}$-norm of the martingale $f$. From (3.9), (3.10),

$$
X_{n}=f_{n}+g_{n}-y \rightarrow X_{\infty}=f_{\infty}+g_{\infty}-y \text { a.e. }
$$

and

$$
Y_{n}=f_{n}-g_{n}+y \rightarrow Y_{\infty}=f_{\infty}-g_{\infty}+y \text { a.e. }
$$

Furthermore,

$$
\begin{aligned}
T_{n} & =E\left(1\left\{\sup _{m \geq 1}\left|g_{m}-y\right| \geq 1\right\} \mid A_{n}\right) \rightarrow E\left(1\left\{\sup _{m \geq 1}\left|g_{m}-y\right|\right\} \mid A_{\infty}\right) \\
& =1\left\{\sup _{m \geq 1}\left|g_{m}-y\right|\right\}=T_{\infty} \quad \text { a.e. }
\end{aligned}
$$

That is, $W_{n} \rightarrow W_{\infty}=\left(X_{\infty}, Y_{\infty}, T_{\infty}\right)$ a.e. By parts (a), (b) and (c) we have

$$
\left|L\left(W_{n}\right)-\right| X_{n}+Y_{n}|| \rightarrow 0 \text { a.e. }
$$

with each term being dominated by $M$. By the Lebesgue dominated convergence theorem,

$$
\lim _{n \rightarrow \infty} E\left(L\left(W_{n}\right)-\left|X_{n}+Y_{n}\right|\right)=E \lim _{n \rightarrow \infty}\left(L\left(W_{n}\right)-\left|X_{n}+Y_{n}\right|\right)=0 .
$$

Therefore

$$
\lim _{n \rightarrow \infty} E L\left(W_{n}\right)=\lim _{n \rightarrow \infty} 2\left\|f_{n}\right\|_{1}=2\|f\|_{1}
$$

because $X_{n}+Y_{n}=2 f_{n}$. By the above calculations and (3.13), we have

$$
\|f\|_{1} \geq \frac{1}{2} L\left(x, y, t^{\prime}\right) \geq \frac{1}{2} L(x, y, t)
$$

since $L(x, y, t)$ is increasing in $t$ for $(x, y)$ fixed.

Part II. We first observe that

$$
f \in \mathcal{N}(x, y, t) \Leftrightarrow f \in \mathcal{N}(y, x, t) \Leftrightarrow-f \in \mathcal{N}(-x,-y, t) .
$$


The first equivalence follows from the observation that if $f \in \mathcal{N}(x, y, t)$ and $v$ and $g$ are associated with $f$ as in the definition then the transform $g^{\prime}$ of $f$ by $v^{\prime}=\left(1,-v_{2},-v_{3}, \ldots\right)$ satisfies

$$
P\left(\sup _{n \geq 1}\left|g_{n}^{\prime}-x\right| \geq 1\right)=P\left(\sup _{n \geq 1}\left|g_{n}-y\right| \geq 1\right) \geq t,
$$

therefore $f \in \mathcal{N}(y, x, t)$. Similar reasoning shows that $\mathcal{N}(y, x, t) \subseteq \mathcal{N}(x, y, t)$. That $f \in \mathcal{N}(x, y, t) \Leftrightarrow-f \in \mathcal{N}(-x,-y, t)$ follows directly from definition. Using (3.3) and (3.14), it is enough to show that (1.7) is sharp for $x>0$ and $-x \leq y \leq x$. Therefore we have six cases: (i) $x \geq 1$ and $y \leq x-2$; (ii) $x \geq 1$ and $x-2<y \leq x$; (iii) $x<1, y \leq 0$ and $(1+y) t \leq x+y$; (iv) $x<1, x+y<(1+y) t,-x \leq y$ and $t \leq(1+y) x-y$; (v) $0 \leq y \leq x<1$ and $t \leq(1-y) x+y$; and our last case (vi) $-x \leq y \leq x$ and $(1-|y|) x+|y|<t$.

It can be seen easily, under cases (i) to (iv), that $L_{1}(x, y, t)=L(x, y, t)$. Furthermore, cases (i) and (ii) above are subcases of (i) and (ii) in Part II of the proof of Theorem 1.1 respectively. Since we assume $y \geq-x$, in cases (iii) and (iv) as $x<1$, therefore (iii) and (iv) are also subcases of (iv) and (v) in Part II of the proof of Theorem 1.1. Therefore (1.7) is sharp for cases (i) to (iv). For case (v), we distinguish the subcases $x \geq t \geq 0$ and $x<t \leq 1$. When $x \geq t \geq 0$, let $f_{1}=((x+y) / 2)[0,1)$,

$$
f_{n}=2^{n-3}(1+y)\left[0, \frac{t}{2^{n-2}}\right)+\frac{1}{2}\left(y+\frac{x-t}{1-t}\right)[t, 1)
$$

for all $n \geq 2$. Now $f$ is a nonnegative martingale and $g$ the transform of $f$ by $v=(1,1,-1,-1,-1, \ldots)$ satisfies

$$
P\left(\sup _{m \geq 1}\left|g_{m}-y\right| \geq 1\right)=t .
$$

That is, $f \in \mathcal{N}(x, y, t)$. Now

$$
\|f\|_{1}=E f_{1}=\frac{x+y}{2}=\frac{1}{2} L(x, y, t)
$$

when $x<t \leq 1$. Here we use the splicing technique. Let

$$
f_{n}^{1}=2^{n-2}(1+y)\left[0,1 / 2^{n-1}\right)
$$

for all $n \geq 1$. For any $\varepsilon>0$ (small), there exists

$$
f^{2} \in \mathcal{N}\left(y, 0, \frac{t-x}{1-x}\right)=\mathcal{N}\left(0, y, \frac{t-x}{1-x}\right)
$$

so that

$$
\left\|f^{2}\right\|_{1}<\varepsilon+\frac{1}{2} L\left(y, 0, \frac{t-x}{1-x}\right)
$$

for $(y, 0,(t-x) /(1-x))$ corresponds to case (iii) above. Without loss of generality, we assume $f^{2}$ is defined on the probability space $([0,1), B, \lambda)$. Define

$$
f_{1} \equiv \frac{x+y}{2}, \quad f_{n+1}(s)= \begin{cases}f_{n}^{1}\left(\frac{s}{x}\right), & 0 \leq s<x \\ f_{n}^{2}\left(\frac{s-x}{1-x}\right), & x \leq s<1\end{cases}
$$


Let $v^{2}=\left(1, v_{2,2}, v_{2,3}, v_{2,4}, \ldots\right)$ be a sequence predictable relative to $f^{2}$ and such that

$$
P\left(\sup _{m \geq 1}\left|g_{m}^{2}-y\right| \geq 1\right) \geq \frac{t-x}{1-x} .
$$

Such a sequence exists because

$$
f^{2} \in \mathcal{N}(0, y,(t-x) /(1-x))
$$

Let $v^{\prime}=\left(1, v_{1,2}, v_{1,3}, \ldots\right)$ where $v_{1, k}=-1$ for all $k \geq 2$. Define

$$
\begin{aligned}
& v_{k+1}(s)=v_{1, k} \quad \text { if } 0 \leq s<x \text {, } \\
& =v_{2, k} \text { if } x \leq s<1 \text {, }
\end{aligned}
$$

for all $k \geq 1$. Then $g$, the transform of $f$ by $v=\left(1, v_{2}, v_{3}, \ldots\right)$, which is predictable relative to $f$, satisfies

$$
P\left(\sup _{n \geq 1}\left|g_{n}-y\right| \geq 1\right) \geq x+(1-x) \frac{t-x}{1-x}=t .
$$

Also $f^{1} \in \mathcal{N}(1, y, 1)$ and $\left\|f^{1}\right\|_{1}=(1+y) / 2$ and

$$
\left\|f^{2}\right\|_{1}<\varepsilon+\frac{1}{2} L\left(y, 0, \frac{t-x}{1-x}\right)=\varepsilon+\frac{y}{2} .
$$

Therefore,

$$
\begin{aligned}
\|f\|_{1} & =\sup _{n \geq 1}\left\|f_{n+1}\right\|_{1}=\lim _{n \rightarrow \infty}\left\|f_{n+1}\right\|_{1} \\
& =\lim _{n \rightarrow \infty} x\left\|f_{n}^{1}\right\|_{1}+(1-x)\left\|f_{n}^{2}\right\|_{1} \\
& \leq \frac{x(1+y)}{2}+\varepsilon+\frac{y(1-x)}{2}=\frac{x+y}{2}+\varepsilon .
\end{aligned}
$$

That is, (1.7) is sharp. This completes case (v).

Case (vi). Now $(t-x) /(1-x)>|y|$ since $(1-|y|) x+|y|<t$. Therefore let

$$
\alpha=\frac{1}{2}\left[\frac{t-x}{1-x}+y\right]>0, \quad \beta=\frac{1}{2}\left[\frac{t-x}{1-x}-y\right]>0,
$$

and

$$
\gamma=1-\alpha-\beta=\frac{1-t}{1-x}>0 .
$$

(Here we assume $t<1$; for $t=1$, see Remark 7.1 of [3].) Define

$$
\begin{aligned}
f_{1} & =\frac{x+y}{2}[0,1) \\
f_{2} & =\frac{1+x}{2}[0, \alpha)-\frac{1-x}{2}[\alpha, \alpha+\beta)+\frac{x}{2}[\alpha+\beta, 1) \\
f_{3} & =2\left[\frac{3-x}{4} \alpha, \alpha\right)-2\left[\alpha+\frac{3+x}{4} \beta, \alpha+\beta\right)+\frac{1}{2}[\alpha+\beta+\gamma(1-x), 1) \\
f_{4} & =2\left[\frac{3-x}{4} \alpha, \alpha\right)-2\left[\alpha+\frac{3+x}{4} \beta, \alpha+\beta\right)+2\left[1-\frac{\gamma x}{4}, 1\right) \\
f_{n} & =f_{4} \text { for all } n \geq 4 \\
v_{1} & =-v_{2}=-v_{4}=[0,1) \\
v_{3} & =[0, \alpha+\beta)-[\alpha+\beta, 1) \\
v_{n} & =[0,1) \text { for all } n \geq 5
\end{aligned}
$$


It is not difficult to verify that $v=\left(1, v_{2}, v_{3}, \ldots\right)$ is predictable relative to $f$ and that $f$ is a martingale. Furthermore,

$$
\left\{\sup _{n \geq 1}\left|g_{n}-y\right| \geq 1\right\}=[0, \alpha+\beta) \cup[\alpha+\beta+\gamma(1-x), 1) .
$$

Therefore,

$$
P\left(\sup _{n \geq 1}\left|g_{n}-y\right| \geq 1\right)=\alpha+\beta+(1-\alpha-\beta)-\gamma(1-x)=t .
$$

Now

$$
\|f\|_{1}=\left\|f_{4}\right\|_{1}=2 \alpha \frac{1+x}{4}+2 \beta \frac{1-x}{4}+2 \frac{\gamma x}{4}=\frac{1}{2}(t+x y)=\frac{1}{2} L(x, y, t) .
$$

This completes the case (vi) and the proof of Theorem 1.2.

In each of the above examples, we observe that $v_{k} \in\{1,-1\}$. Using the proof of Lemma 2.1 in [3], we have the remark immediately after Theorem 1.2.

\section{Properties of the functions $L$ and $L_{1}$.}

THEOREM 4.1. The function $L$ is the greatest function $u$ on $\bar{\Omega}$ such that the map $(x, t) \mapsto u(x, y, t)$ is convex on the section of $\bar{\Omega}$ determined by $y$,

the map $(y, t) \mapsto u(x, y, t)$ is convex on the section of $\bar{\Omega}$ determined by $x$,

PROOF. Suppose $u$ is any function satisfying (4.1) to (4.4). We want to show that

$$
u(x, y, t) \leq L(x, y, t) \text { on } \bar{\Omega} .
$$

In the proof of (3.8), we show that $\psi(s)=L(x+h s, y+k s, t+r s)$ is convex in $s$. If we set $k=0$, the convexity of $\psi(s)$ is equivalent to saying that the map $(x, t) \mapsto L(x, y, t)$ is convex, so $L$ satisfies (4.1). The function $L$ also satisfies (4.2) by symmetry in (3.3).

Without loss of generality, we may assume

$$
u(x, y, t)=u(-x,-y, t)=u(y, x, t)=u(-y,-x, t) .
$$

Otherwise replace $u(x, y, t)$ by $\tilde{u}(x, y, t)$ : the maximum of $u(x, y, t), u(-x,-y, t)$, $u(y, x, t)$ and $u(-y,-x, t)$. The function $\tilde{u}$ satisfies (4.1) to (4.4).

With the symmetry conditions and continuity, to show (4.5), it suffices to show (4.5) for $x>0$ and $-x<y<x$.

(i) If $x \geq 1$, then for $0 \leq t \leq 1$,

$$
u(x, y, t) \leq t u(x, y, 1)+(1-t) u(x, y, 0) \leq|x+y|=L(x, y, t) .
$$


We assume $0<x<1$ below. If $t=0,(4.5)$ is immediate from (4.3),

$$
\begin{aligned}
u(x, y, 1) & \leq \frac{1-y}{2} u(x,-1,1)+\frac{1+y}{2} u(x, 1,1) \\
& \leq \frac{(1-y)(1-x)}{2}+\frac{(1+y)(1+x)}{2} \text { by }(4.4) \\
& =1+x y=L(x, y, 1) .
\end{aligned}
$$

Therefore (4.5) holds in this case.

So we further asssume $0<t<1$.

(ii) If $y \leq 0$ and $x+y \geq(1+y) t$, then

$$
\begin{aligned}
u(x, y, t) & =u\left(t \cdot 1+(1-t) \frac{x-t}{1-t}, y, t \cdot 1+(1-t) \cdot 0\right) \\
& \leq t u(1, y, 1)+(1-t) u\left(\frac{x-t}{1-t}, y, 0\right) \\
& \leq t(1+y)+(1-t)\left|\frac{x-t}{1-t}+y\right| \\
& =t(1+y)+|x+y-(1+y) t| \\
& =x+y=L(x, y, t) .
\end{aligned}
$$

(iii) If $y \geq t>0$ and $(1-y) x+y \geq t$, then

$$
\begin{aligned}
u(x, y, t) & =u\left(x, t \cdot 1+(1-t) \cdot \frac{y-t}{1-t}, t \cdot 1+(1-t) \cdot 0\right) \\
& \leq t u(x, 1,1)+(1-t) u\left(x, \frac{y-t}{1-t}, 0\right) \\
& \leq t(1+x)+(1-t)\left|x+\frac{y-t}{1-t}\right| \\
& =x+y \quad \text { since } x>0 \text { and } y-t \geq 0 \\
& =L(x, y, t) .
\end{aligned}
$$

(iv) If $1>t>y>0$ and $(1-y) x+y \geq t$, then

$$
\begin{aligned}
u(x, y, t) & =u\left(x, y \cdot 1+(1-y) \cdot 0, y \cdot 1+(1-y) \cdot \frac{t-y}{1-y}\right) \\
& \leq y u(x, 1,1)+(1-y) L\left(x, 0, \frac{t-y}{1-y}\right) .
\end{aligned}
$$

Now $(x, 0,(t-y) /(1-y))$ is as in case (ii), therefore

$$
u(x, y, t) \leq y(1+x)+(1-y) x=x+y=L(x, y, t) .
$$

(v) In this case $t \geq(1-|y|) x+|y|$. Let

$$
\alpha=\frac{1}{2}\left[\frac{t-x}{1-x}+y\right], \quad \beta=\frac{1}{2}\left[\frac{t-x}{1-x}-y\right] .
$$

Since $t \geq(1-|y|) x+|y|$, we have $(t-x) /(1-x) \geq|y|$, that is, $\alpha, \beta \geq 0$. Also $\alpha+\beta=(t-x) /(1-x)<1$. Note that

$$
\begin{aligned}
u(x, y, t) & =u(x, \alpha \cdot 1+\beta \cdot(-1)+(1-\alpha-\beta) \cdot 0, \alpha \cdot 1+\beta \cdot 1+(1-\alpha-\beta) x) \\
& \leq \alpha u(x, 1,1)+\beta u(x,-1,1)+(1-\alpha-\beta) u(x, 0, x) \\
& \leq \alpha(1+x)+\beta(1-x)+(1-\alpha-\beta) x
\end{aligned}
$$


since $(x, 0, x)$ is as in case (ii). We have

$$
u(x, y, t) \leq(\alpha+\beta)(1-x)+(\alpha-\beta) x+x=t+x y=L(x, y, t) .
$$

(vi) We now consider the remaining case: $y \leq 0, x+y<t(1+y)$ and $t<$ $(1+y) x-y$. From the second inequality, we have $0<(1-t)(1+y) /(1-x)<1$, and from the third inequality we have $(1+y)(1-x) /(1-t)>1$.

Let

$$
\lambda=1-\left[\frac{(1+y)(1-t)}{1-x}\right]^{1 / 2} \text { and } Y=\left[\frac{(1-x)(1+y)}{1-t}\right]^{1 / 2}-1,
$$

therefore

$$
u(x, y, t)=u\left(x, \lambda(-1)+(1-\lambda) Y, \lambda \cdot 1+(1-\lambda) \frac{Y+x}{1+Y}\right)
$$

since

$$
\lambda(-1)+(1-\lambda) Y=-1+(1-\lambda)(1+Y)=y,
$$

and

$$
\lambda+(1-\lambda) \frac{Y+x}{1+Y}=1-(1-\lambda) \frac{1-x}{1+Y}=t .
$$

Therefore,

$$
u(x, y, t) \leq \lambda u(x,-1,1)+(1-\lambda) u\left(x, Y, \frac{Y+x}{1+Y}\right) .
$$

It can be checked that $(1-Y) x+Y \geq(Y+x) /(1+Y)$, that is, $(x, Y,(Y+x) /(1+x))$ belongs to case (iii). So

$$
\begin{aligned}
u(x, y, t) & \leq \lambda(1-x)+(1-\lambda)(x+Y) \\
& =(\lambda-1+1)(1-x)+(1-\lambda)(1+Y-(1-x)) \\
& =1-x-2(1-\lambda)(1-x)+(1-\lambda)(1+Y) \\
& =2+y-x-2[(1-x)(1+y)(1-t)]^{1 / 2} \\
& =L(x, y, t) .
\end{aligned}
$$

This completes the proof of Theorem 4.1.

We have a similar theorem.

THEOREM 4.2. The function $L_{1}$ is the greatest function $w: D \rightarrow \mathbf{R}$ satisfying (4.1) to (4.3) and (4.4') where

$$
w(x, y, 1) \leq|x+y| \quad \text { if } x \geq 1 \text { or } y \leq-1 .
$$

PROOF. From the observation in the last paragraph of Part I of the proof of Theorem 1.1, we have

$$
L_{1}(x, y, t)=|x+y| \vee\left\{2+y-x-2\left[(1-x)^{+}(1+y)^{+}(1-t)\right]^{1 / 2}\right\} .
$$

Now

$$
2+y-x-2\left[(1-x)^{+}(1+y)^{+}(1-t)\right]^{1 / 2}
$$

satisfies (4.1) and (4.2) on $\{(x, y, t) \in D: x \leq 1$ and $y \geq-1\}$ by differentiation. Indeed,

$$
\frac{\partial^{2} L_{1}}{\partial x^{2}}, \frac{\partial^{2} L_{1}}{\partial y^{2}}, \frac{\partial^{2} L_{1}}{\partial t^{2}} \geq 0
$$


and

$$
\frac{\partial^{2} L_{1}}{\partial x^{2}} \frac{\partial^{2} L_{1}}{\partial t^{2}}-\left(\frac{\partial^{2} L_{1}}{\partial x \partial t}\right)^{2}=\frac{\partial^{2} L_{1}}{\partial y^{2}} \frac{\partial^{2} L_{1}}{\partial t^{2}}-\left(\frac{\partial^{2} L_{1}}{\partial y \partial t}\right)^{2}=0
$$

Then it is not difficult to see that $L_{1}$ satisfies (4.1) and (4.2). To show that $L_{1}$ is maximal, we may assume that $w$ satisfies the symmetry condition (3.1), otherwise we replace $w(x, y, t)$ by the maximum of $w(x, y, t)$ and $w(-y,-x, t)$. Hence it is sufficient to show

$$
w(x, y, t) \leq L_{1}(x, y, t) \text { for } 0<t<1 \text { and } x+y \geq 0
$$

When $x \geq 1$ or $y \leq-1$, then

$$
w(x, y, t) \leq t w(x, y, 1)+(1-t) w(x, y, 0) \leq x+y=L_{1}(x, y, t)
$$

We first show that, for $y \geq-1$,

$$
w(-y, y, t) \leq 2(1+y)\left[1-(1-t)^{1 / 2}\right]=L_{1}(-y, y, t)
$$

Let $\alpha=(1-t)^{1 / 2}$. Then $-y=\alpha[-(y+1) / \alpha+1]+(1-\alpha) \cdot 1$ so

$$
\begin{aligned}
w(-y, y, t) \leq & \alpha w\left(-\frac{1+y}{\alpha}+1, y, 1-(1-t)^{1 / 2}\right)+(1-\alpha) w(1, y, 1) \\
\leq & \alpha\left\{\alpha w\left(-\frac{1+y}{\alpha}+1, \frac{1+y}{\alpha}-1,0\right)+(1-\alpha) w\left(-\frac{1+y}{\alpha}+1,-1,1\right)\right\} \\
& +(1-\alpha) w(1, y, 1) \\
\leq & \alpha(1-\alpha) \frac{1+y}{\alpha}+(1-\alpha)(1+y)=2(1+y)\left[1-(1-t)^{1 / 2}\right] \\
= & L_{1}(-y, y, t) .
\end{aligned}
$$

From now on, we assume $x+y>0, x<1, y>-1$ and $0<t<1$.

(i) If $(x, y, t) \in D_{1}$, then

$$
\begin{aligned}
w(x, y, t) & =w\left(t \cdot 1+(1-t)\left(1-\frac{1-x}{1-t}\right), y, t \cdot 1+(1-t) \cdot 0\right) \\
& \leq t w(1, y, 1)+(1-t) w\left(1-\frac{1-x}{1-t}, y, 0\right) \\
& \leq t(1+y)+(1-t)\left|1-\frac{1-x}{1-t}+y\right| \\
& =x+y, \quad \text { since } 1+y-\frac{1-x}{1-t}>0 \text { on } D_{1} \\
& \leq L_{1}(x, y, t) .
\end{aligned}
$$


(ii) If $(x, y, t) \in D_{2}$, then $1-t<(1-x) /(1+y)$ and

$$
\begin{aligned}
w(x, y, t) & =w\left(\frac{x+y}{1+y} \cdot 1+\frac{1-x}{1+y}(-y), y, \frac{x+y}{1+y} \cdot 1+\frac{1-x}{1+y}\left(1-\frac{(1-t)(1+y)}{1-x}\right)\right) \\
& \leq \frac{x+y}{1+y} w(1, y, 1)+\frac{1-x}{1+y} w\left(-y, y, 1-\frac{(1-t)(1+y)}{1-x}\right) \\
& \leq x+y+\frac{1-x}{1+y} \cdot 2(1+y)\left[1-\left[\frac{(1-t)(1+y)}{1-x}\right]^{1 / 2}\right] \quad \text { by }(4.7) \\
& =2+y-x-2[(1-x)(1+y)(1-t)]^{1 / 2} \\
& \leq L_{1}(x, y, t) .
\end{aligned}
$$

This completes the proof of Theorem 4.2.

In the remainder of this section, we describe how we obtain the functions $L(x, y, t)$ and $L_{1}(x, y, t)$.

The author first obtained the explicit form of $L_{1}(x, y, t)$ by finding the greatest function $w:(-\infty, 1] \times[-1, \infty) \times[0,1] \rightarrow \mathbf{R}$ that satisfies the following partial differential inequalities:

$$
\begin{aligned}
& w_{x x} \geq 0 \\
& w_{y y} \geq 0 \\
& w_{t t} \geq 0 \\
& w_{x x} w_{t t}-w_{x t}^{2} \geq 0,
\end{aligned}
$$

and

$$
w_{y y} w_{t t}-w_{y t}^{2} \geq 0
$$

on any neighborhood on which $w$ is twice continuously differentiable, $w$ satisfying the boundary conditions

$$
\begin{array}{ll}
w(x, y, 0) \leq|x+y| & \text { if } x \leq 1 \text { and } y \geq-1 \\
w(x, y, 1) \leq|x+y| & \text { if } x=1 \text { or } y=-1
\end{array}
$$

Then the author solves the problem of finding the greatest function $u:[-1,1] \times$ $[-1,1] \times[0,1] \rightarrow \mathbf{R}$ satisfying (4.8) to (4.12) and with boundary conditions

$$
\begin{array}{ll}
u(x, y, 0) \leq|x+y| & \text { if }|x| \vee|y| \leq 1 \\
u(x, y, 1) \leq|x+y| & \text { if }|x| \vee|y|=1
\end{array}
$$

If $w:(-\infty, 1] \times[-1, \infty) \times[0,1] \rightarrow \mathbf{R}$ satisfies $(4.8)$ to $(4.14)$, then $\tilde{w}:[0, \infty) \times$ $[0, \infty) \times[0,1] \rightarrow \mathbf{R}$ defined by

$$
\tilde{w}(x, y, t)=w(1-x, y-1, t)
$$

will satisfy (4.8) to (4.14) with boundary conditions

$$
\begin{array}{ll}
\tilde{w}(x, y, 0) \leq|x-y| & \text { if } x \geq 0 \text { and } y \geq 0, \\
\tilde{w}(x, y, 1)=|y-x| & \text { if } x=0 \text { or } y=0 .
\end{array}
$$

Suppose there exists a function $\tilde{w}$ satisfying (4.8) to (4.12) and (4.15) to (4.16), then $(1 / \lambda) \tilde{w}(\lambda x, \lambda y, t)$ will also satisfy (4.8) to (4.12), (4.15) and (4.16) for all $\lambda>0$. 
Therefore, the greatest function $\tilde{L}_{1}$ that satisfies (4.8) to (4.12), (4.15) and (4.16) will have the positive homogeneity property:

$$
\tilde{L}_{1}(\lambda x, \lambda y, t)=\lambda \tilde{L}_{1}(x, y, t), \quad \lambda>0 .
$$

Define $F:[0, \infty) \times[0,1] \rightarrow \mathbf{R}$ by $F(s, t)=\tilde{L}_{1}(s, 1, t)$. Therefore, for $y>0$,

$$
\tilde{L}_{1}(x, y, t)=y F(x / y, t) \text {. }
$$

By this relationship, we are led to consider

$$
\begin{aligned}
& F_{s s} \geq 0 \\
& F_{t t} \geq 0 \\
& F_{s s} F_{t t}-F_{s t}^{2} \geq 0
\end{aligned}
$$

and

$$
s^{2} F_{s s} F_{t t}-\left[F_{t}-s F_{s t}\right]^{2} \geq 0
$$

and boundary conditions

$$
\begin{aligned}
& F(s, 0) \leq|1-s|, \quad s \geq 0, \\
& F(0,1) \leq 1 .
\end{aligned}
$$

A solution to (4.18) to (4.23) is found, namely: let $F:[0, \infty) \times[0,1] \rightarrow \mathbf{R}$ be the continuous function satisfying $F(s, t)=s F(1 / s, t), s>0$, and

$$
F(s, t)=1+s-2[s(1-t)]^{1 / 2}, \quad 0<s \leq 1 .
$$

Indeed $F$ satisfies (4.20), (4.21) and (4.23) with equality and (4.18), (4.19) and (4.22) with strict inequality.

Thus $L_{1}(x, y, t)$ is obtained.

\section{Proof of Lemma 3.1.}

PROOF OF LEMMA 3.1. (a). If $|x| \vee|y| \geq 1$, then $L(x, y, t)-|x+y|=0$. Since $L(x, y, t)-|x+y|$ is continuous, we must have $|L(x, y, t)-| x+y|| \leq M$ on $[-1,1] \times[-1,1] \times[0,1]$ for some positive real number $M$.

(b) and (c). These cases are obvious.

(d). To show that (3.8) holds we observe that

$$
\psi(s)=L(x+h s, y+k s, t+r s)
$$

is continuous in $s$ for those $s$ such that $(x+h s, y+k s, t+r s) \in \mathbf{R} \times \mathbf{R} \times[0,1]$. We will first show that $\psi(s)$ is convex and then deduce (3.8) from the convexity of $\psi$.

To this end, it suffices to show that $\psi$ is locally convex in $s$ because $\psi$ is continuous. Define, for $1 \leq i \leq 5, Q_{i}=\Omega_{i} \rightarrow \mathbf{R}$ by

$$
\begin{aligned}
& Q_{1}(x, y, t)=x+y \quad \text { on } \Omega_{1} \text {, } \\
& Q_{2}(x, y, t)=2+y-x-2[(1-x)(1+y)(1-t)]^{1 / 2} \text { on } \Omega_{2} \text {, } \\
& Q_{3}(x, y, t)=Q_{1}(-x,-y, t) \quad \text { on } \Omega_{3} \text {, } \\
& Q_{4}(x, y, t)=Q_{2}(-x,-y, t) \quad \text { on } \Omega_{4} \text {, } \\
& Q_{5}(x, y, t)=t+x y \quad \text { on } \Omega_{5} \text {. }
\end{aligned}
$$

And define, for all $1 \leq i \leq 5$,

$$
\psi_{i}(s)=Q_{i}(x+h s, y+k s, t+r s)
$$


for those $s$ such that $(x+h s, y+k s, t+r s) \in \Omega_{i}$.

For each $1 \leq i \leq 5, \psi_{i}$ is convex. In fact $\psi_{i}$ is twice differentiable and $\psi_{1}^{\prime \prime}(s)=$ $\psi_{3}^{\prime \prime}(s)=0$. Now

$$
\psi_{2}(s)=2+y-x+(k-h) s-2[M(s)]^{1 / 2}
$$

where

$$
M(s)=(1-x-h s)(1+y+k s)(1-t-r s) .
$$

Therefore

$$
\psi_{2}^{\prime \prime}(s)=\frac{\left[M^{\prime}(s)\right]^{2}-2 M^{\prime \prime}(s) M(s)}{2[M(s)]^{3 / 2}} .
$$

Now

$$
\begin{aligned}
& {\left[M^{\prime}(s)\right]^{2}-2 M(s) M^{\prime \prime}(s)=4 h k(1-x-h s)(1+y+k s)(1-t-r s)^{2}} \\
& +[h(1+y+k s)(1-t-r s)-k(1-x-h s)(1-t-r s) \\
& -r(1-x-h s)(1+y+k s)]^{2} \geq 0
\end{aligned}
$$

since $h k \geq 0$. Therefore $\psi_{2}$ is convex. Similarly, $\psi_{4}^{\prime \prime}$ is nonnegative.

Since $\psi_{5}(s)=t+r s+(x+h s)(y+k s)$ then $\psi_{5}^{\prime \prime}(s)=2 h k \geq 0$.

Therefore to establish the local convexity of $\psi$, it is enough to assume that $(x, y, t) \in \bigcup_{i=1}^{5} \partial \Omega_{i}$. By the symmetry conditions (1.10), we may assume $x>0$ and $-x \leq y \leq x$. We have the following cases: (i) $0<x<1, y>0,(1-y) x+y=t$; (ii) $0<x<1,(1+y) x-y=t, y<0$; (iii) $y<0$, $(1+y) t=x+y$; (iv) $x \geq 1$, $y=-x$ and (v) $x=t, y=0$.

To check local convexity of $\psi$ for these cases, we only need to show $\psi^{\prime}(0+) \geq$ $\psi^{\prime}(0-)$ because $\psi^{\prime \prime}(s)>0$ for $s \in(-\delta, 0) \cup(0, \delta)$ where $\delta$ depends on $(x, y, t)$ and $(h, k, r)$ and is sufficiently small and positive.

We may further assume that $h \geq 0$ and $k \geq 0$ since the convexity of the function $L(x+h s, y+k s, t+r s)$ in $s$ will imply the convexity of the function $L(-x+(-h) s,-y+(-k) s, t+r s)$ in $s$.

For case (i), suppose $r<h(1-y)+k(1-x)$. Choose $\delta>0$ so small that $r+h k \delta \leq h(1-y)+k(1-x)$, then

$$
\psi(s)= \begin{cases}x+y+(h+k) s, & 0 \leq s<\delta \\ t+r s+(x+h s)(y+k s), & -\delta<s \leq 0\end{cases}
$$

so

$$
\psi^{\prime}(0+)=h+k \geq r+h y+k x=\psi^{\prime}(0-) .
$$

Suppose $r>h(1-y)+k(1-x)$ and choose $\delta>0$ such that $r \geq h(1-y)+$ $k(1-x)+h k \delta$. Then

$$
\psi(s)= \begin{cases}t+r s+(x+h s)(y+k s), & 0 \leq s<\delta \\ x+y+(h+k) s, & -\delta<s \leq 0\end{cases}
$$

so

$$
\psi^{\prime}(0+)=r+h y+k x \geq h+k=\psi^{\prime}(0-) .
$$

When $r=h(1-y)+k(1-x)$, choose $\delta>0$ such that $0 \leq t+r s \leq 1$; then

$$
\psi(s)=t+r s+(x+h s)(y+k s)
$$

which is convex in $s$. This finishes case (i). 
For case (ii), $\psi^{\prime}(0)$ exists because $L_{x}, L_{y}$ and $L_{t}$ are continuous at $(x, y, t)$ when $0<x<1, y<0$ and $(1+y) x-y=t$.

For case (iii), suppose $r(1+y)<h+k(1-t)$. Choose $\delta>0$ so that $r(1+y)+h k \delta \leq$ $h+k(1-t)$, then

$$
\psi(s)= \begin{cases}x+y+(h+k) s, & 0 \leq s<\delta \\ 2+y-x+(k-h) s-2[M(s)]^{1 / 2}, & -\delta<s \leq 0\end{cases}
$$

Now

$$
\psi^{\prime}(0-)=k t+r(1+y) \leq h+k=\psi^{\prime}(0+) .
$$

Suppose $r(1+y)>h+k(1-t)$ and choose $\delta>0$ such that $r(1+y) \geq h+$ $k(1-t)+k r \delta$; then

$$
\psi(s)= \begin{cases}2+y-x+(k-h) s-2[M(s)]^{1 / 2}, & 0 \leq s<\delta, \\ x+y+(k+h) s, & -\delta<s \leq 0,\end{cases}
$$

and

$$
\psi^{\prime}(0+)=k t+r(1+y) \geq h+k=\psi^{\prime}(0-) .
$$

When $r(1+y)=h+k(1-t)$,

$$
\psi(s)=2+y-x+(k-h) s-2[M(s)]^{1 / 2}
$$

is convex in $s$.

For case (iv),

$$
\psi(s)= \begin{cases}(h+k) s, & 0 \leq s<\delta \\ -(h+k) s, & -\delta<s \leq 0,\end{cases}
$$

so $\psi^{\prime}(0+) \geq \psi^{\prime}(0-)$. Case $(\mathrm{v})$ is treated similarly and this completes the proof of convexity of $\psi$.

Let $\varphi_{i}: \mathbf{R} \times \mathbf{R} \times[0,1] \rightarrow \mathbf{R}$ satisfy the following relationships

$$
\begin{aligned}
& \varphi_{1}(x, y, t)=-\varphi_{1}(-x,-y, t) \\
& \varphi_{1}(x, y, t)=\varphi_{2}(y, x, t), \\
& \varphi_{3}(x, y, t)=\varphi_{3}(-x,-y, t)=\varphi_{3}(y, x, t) .
\end{aligned}
$$

If $0<t<1$, define

If $0<t<1$,

$$
\begin{gathered}
\varphi_{1}(x, y, t)= \begin{cases}L_{x}(x, y, t) & \text { on } \bigcup_{i=1}^{5} \Omega_{i}, \\
y & \text { if } y>0 \text { and }(1-y) x+y=t \\
-1 & \text { if }(\mathrm{i}) x=-y \text { and } x \geq 1 \text { or }\end{cases} \\
\varphi_{1}(x, y, 0)= \begin{cases}1 & \text { ii) } 0 \leq x \leq 1 \text { and } x+y=(1+y) t \\
-1 & \text { if } x+y \leq 0\end{cases} \\
\varphi_{1}(x, y, 1)= \begin{cases}1 & \text { if } x+y>0, x \vee y>1 \\
y & \text { if }|x| \vee|y|<1 \\
-1 & \text { otherwise. }\end{cases}
\end{gathered}
$$

$$
\begin{aligned}
& \varphi_{3}(x, y, t)= \begin{cases}L_{t}(x, y, t) & \text { on } \bigcup_{i=1}^{5} \Omega_{i}, \\
0 & \text { otherwise, }\end{cases} \\
& \varphi_{3}(x, y, 0)=0 \text { for all }(x, y) \in \mathbf{R}^{2}, \\
& \varphi_{3}(x, y, 1)= \begin{cases}1 & \text { if }|x| \vee|y|<1, \\
0 & \text { if }|x| \vee|y| \geq 1\end{cases}
\end{aligned}
$$


The functions $\varphi_{i}, 1 \leq i \leq 3$, are well defined and are bounded in absolute value. We now deduce (3.8). If $s>\delta>0$, then

$$
\frac{\psi(s)-\psi(0)}{s} \geq \frac{\psi(\delta)-\psi(0)}{\delta} .
$$

If $s<\delta<0$, then

$$
\frac{\psi(s)-\psi(0)}{s} \leq \frac{\psi(\delta)-\psi(0)}{\delta} .
$$

In either case, if $|s|>|\delta|>0$ and $s \delta>0$, we have

$$
\psi(s) \geq \psi(0)+s \frac{\psi(\delta)-\psi(0)}{\delta} .
$$

Therefore we have

$$
\psi(s) \geq \psi(0)+s \liminf _{\substack{\delta \rightarrow 0 \\ s \delta \geq 0}} \frac{\psi(\delta)-\psi(0)}{\delta} .
$$

Recalling the definition of $\psi$, we see that the above inequality becomes

$$
\begin{aligned}
& L(x+h s, y+k s, t+r s) \\
& \quad \geq L(x, y, t)+s \liminf _{\substack{\delta \rightarrow 0 \\
s \delta \geq 0}} \frac{L(x+h \delta, y+k \delta, t+r \delta)-L(x, y, t)}{\delta} .
\end{aligned}
$$

Therefore (3.8) follows if we have

$$
\begin{aligned}
& \liminf _{\delta \rightarrow 0+} \frac{L(x+h \delta, y+k \delta, t+r \delta)-L(x, y, t)}{\delta} \\
& \geq h \varphi_{1}(x, y, t)+k \varphi_{2}(x, y, t)+r \varphi_{3}(x, y, t), \\
& \liminf _{\delta \rightarrow 0-} \frac{L(x+h \delta, y+k \delta, t+r \delta)-L(x, y, t)}{\delta} \\
& \leq h \varphi_{1}(x, y, t)+k \varphi_{2}(x, y, t)+r \varphi_{3}(x, y, t) .
\end{aligned}
$$

As $L$ coincides with $Q_{i}$ on $\Omega_{i}$ for each $1 \leq i \leq 5$ and $Q_{i}$ is twice differentiable, therefore $L$ is twice differentiable on $\bigcup_{i=1}^{5} \Omega_{i}$. It is not difficult to check that on $\bigcup_{i=1}^{5} \Omega_{i}, \varphi_{1}(x, y, t)=L_{x}(x, y, t), \varphi_{2}(x, y, t)=L_{y}(x, y, t)$ and $\varphi_{3}(x, y, t)=$ $L_{t}(x, y, t)$. And thus, on $\bigcup_{i=1}^{5} \Omega_{i}$, we have equality in (5.4) and (5.5) for both sides are equal to $h L_{x}(x, y, t)+k L_{y}(x, y, t)+r L_{t}(x, y, t)$. Furthermore, when (i) $0<t<1, x<0, y>0$ and $-(1-y) x+y=t$ or (ii) $0<t<1, x>0, y<0$ and $(1+y) x-y=t, L$ has continuous partial derivatives $L_{x}, L_{y}$ and $L_{t}$, and hence there are equalities in (5.4) and (5.5).

We will show (5.4). Then (5.5) is derived from it.

We first assume $0<t<1$.

(i) $0<x \leq 1,0<y \leq 1$ and $(1-y) x+y=t$. Suppose $r<h(1-y)+k(1-x)$; then for small $\delta>0$, we have $(x+h \delta, y+k \delta, t+r \delta) \in \Omega_{1}^{\prime \prime}$, therefore

$$
\begin{aligned}
\lim _{\delta \rightarrow 0+} \frac{L(x+h \delta, y+k \delta, t+r \delta)-L(x, y, t)}{\delta} & =h+k \geq h y+k \cdot x+r \cdot 0 \\
= & h \varphi_{1}(x, y, t)+k \varphi_{2}(x, y, t)+r \varphi_{3}(x, y, t) .
\end{aligned}
$$


Suppose $r \geq h(1-y)+k(1-x)>0$; then for $\delta>0$ small, we have $(x+h \delta, y+$ $k \delta, t+r \delta) \in \Omega_{5}$, therefore

$$
\begin{aligned}
\lim _{\delta \rightarrow 0+} & \frac{L(x+h \delta, y+k \delta, t+r \delta)-L(x, y, t)}{\delta} \\
\quad= & \lim _{\delta \rightarrow 0+} \frac{t+r \delta+(x+h \delta)(y+k \delta)-(t+x y)}{\delta} \\
\quad & r+h y+k x \geq h \varphi_{1}(x, y, t)+k \varphi_{2}(x, y, t)+r \varphi_{3}(x, y, t) .
\end{aligned}
$$

(ii) $-1<y<0$ and $x+y=(1+y) t$. Suppose $r(1+y)<h+k(1-t)$; then $(x+h \delta, y+k \delta, t+r \delta) \in \Omega_{1}^{\prime \prime \prime}$ for small $\delta>0$. Then the limit in (5.4)

$$
=h+k \geq h \cdot 0+k \cdot t+r \cdot 0 \geq h \varphi_{1}(x, y, t)+k \varphi_{2}(x, y, t)+r \varphi_{3}(x, y, t) .
$$

Suppose $r(1+y) \geq h+k(1-t) \geq 0$; then for small $\delta>0,(x+h \delta, y+k \delta, t+r \delta) \in \Omega_{2}$, therefore the limit in (5.4)

$$
\begin{aligned}
= & \lim _{\delta \rightarrow 0+} \frac{1}{\delta}\left\{2+y-x+(k-h) \delta-2[(1-x-h \delta)(1+y+k \delta)(1-t-r \delta)]^{1 / 2}\right. \\
& \left.-\left(2+y-x-2[(1-x)(1+y)(1-t)]^{1 / 2}\right)\right\} \\
= & k-h \\
& +2 \lim _{\delta \rightarrow 0} \frac{[(1-x)(1+y)(1-t)]^{1 / 2}-[(1-x-h \delta)(1+y+k \delta)(1-t-r \delta)]^{1 / 2}}{\delta} \\
= & k-h+\frac{h(1+y)(1-t)-k(1-x)(1-t)+r(1-x)(1+y)}{[(1-x)(1+y)(1-t)]^{1 / 2}} \\
= & k-h+h-k(1-t)+r(1+y), \quad \text { since }(1+y)(1-t)=1-x, \\
= & k t+r(1+y) \geq \text { the right side of }(5.4) .
\end{aligned}
$$

(iii) $-1<x<0$ and $x+y=(1+x) t$. Similar calculations as in (ii) shows (5.4).

(iv) $x+y=0$ and $y \geq 1$. Then $(x+h s, y+k s, t+r s) \in \Omega_{1}^{\prime}$, otherwise $h=k=0$ and (5.4) holds for both sides are equal to 0 , hence

$$
\begin{aligned}
\lim _{\delta \rightarrow 0+} \frac{L(x+h \delta, y+k \delta, t+r \delta)-L(x, y, t)}{\delta}=h+k \\
\geq-h-k+r \cdot 0=h \varphi_{1}(x, y, t)+k \varphi_{2}(x, y, t)+r \varphi_{3}(x, y, t)
\end{aligned}
$$

(v) $x+y=0$ and $x \geq 1$. This case is also similarly handled as in (iv) above.

(vi) $0 \leq x \leq 1$ and $x+y=-(1-x) t$.

(vii) $-1 \leq x<0,-1 \leq y<0$ and $-(1+y) x-y=t$.

(viii) $-1 \leq x \leq 0$ and $-(x+y)=(1-y) t$.

These cases are treated as in the above calculations.

We perform similar calculations for $t=0, t=1$ and $(x, y) \in \mathbf{R}^{2}$ to show (5.4). This completes the proof of (5.4). 
We deduce (5.5) from (5.4):

$$
\begin{aligned}
\lim _{\delta \rightarrow 0-} & \frac{L(x+h \delta, y+k \delta, t+r \delta)-L(x, y, t)}{\delta} \\
& =-\lim _{\varepsilon \rightarrow 0+} \frac{L(x-h \varepsilon, y-k \varepsilon, t-r \varepsilon)-L(x, y, t)}{\varepsilon} \\
& =-\lim _{\varepsilon \rightarrow 0+} \frac{L(-x+h \varepsilon,-y+k \varepsilon, t+(-r) \varepsilon)-L(-x,-y, t)}{\varepsilon} \quad \text { by }(3.3) \\
& \leq-\left\{h \varphi_{1}(-x,-y, t)+k \varphi_{2}(-x,-y, t)-r \varphi_{3}(-x,-y, t)\right\} \quad \text { by }(5.4) \\
& =h \varphi_{1}(x, y, t)+k \varphi_{2}(x, y, t)+r \varphi_{3}(x, y, t) \quad \text { by }(5.2)
\end{aligned}
$$

which is (5.5). This completes the proof of Lemma 3.1.

\section{REFERENCES}

1. D. L. Burkholder, Martingale transforms, Ann. Math. Statist. 37 (1966), 1494-1504.

2. _ A geometrical characterization of Banach spaces in which martingale difference sequences are unconditional, Ann. Probab. 9 (1981), 997-1011.

3. __ Boundary value problems and sharp inequalities for martingale transforms, Ann. Probab. 12 (1984), 647-702.

4. __ An extension of a classical martingale inequality, Prob. Theory and Harmonic Analysis (J.-A. Chao and Worjczyński, eds.), Marcel Dekker, New York, 1986, pp. 21-30.

5. J. L. Doob, Regularity properties of certain families of chance variables, Trans. Amer. Math. Soc. 47 (1940), 455-486.

6. J. Ville, Étude critique de la notion de collectif, Gauthier-Villars, Paris, 1939.

Department of Mathematics, National University of Singapore, Lower KENT RIDGE ROAD, SINGAPORE 0511 